\title{
Helical damping and dynamical critical skin effect in open quantum systems
}

\author{
Chun-Hui Liu, ${ }^{1,2}$ Kai Zhang $\odot,{ }^{1,2}$ Zhesen Yang, ${ }^{1,2}$ and Shu Chen $\odot^{1,2,3, *}$ \\ ${ }^{1}$ Beijing National Laboratory for Condensed Matter Physics, Institute of Physics, Chinese Academy of Sciences, Beijing 100190, China \\ ${ }^{2}$ School of Physical Sciences, University of Chinese Academy of Sciences, Beijing 100049, China \\ ${ }^{3}$ Yangtze River Delta Physics Research Center, Liyang, Jiangsu 213300, China
}

(Received 11 May 2020; accepted 6 October 2020; published 30 October 2020)

\begin{abstract}
Non-Hermitian skin effect and critical skin effect are unique features of non-Hermitian systems. In this work, we explore novel phenomena associated with the $\mathbb{Z}_{2}$ skin effect and critical skin effect in open quantum systems. For the open system with the corresponding damping matrix exhibiting $\mathbb{Z}_{2}$ skin effect, we identify the existence of helical damping, characterized by the evolution of relative particle number with exponentially and algebraically decreasing intervals distinguished by sharp wave fronts with opposite propagation directions. When the time-reversal symmetry is broken by adding perturbations, we find the occurrence of dynamical critical skin effect, which causes the disappearance of the helical damping in the thermodynamic limit although it can survive in small size systems. We also demonstrate the existence of anomalous critical skin effect when we couple two identical systems with $\mathbb{Z}_{2}$ skin effect. With the help of non-Bloch band theory, we unveil that the change of generalized Brillouin zone equation is the origin of critical skin effect.
\end{abstract}

DOI: 10.1103/PhysRevResearch.2.043167

\section{INTRODUCTION}

Non-Hermitian systems are attracting growing attention as they demonstrate some novel properties without Hermitian counterparts [1-21] and many physical problems in photonic systems, electrical systems and open quantum systems can be converted to non-Hermitian Hamiltonian problems [22-28]. In general, a Markovian open quantum system can be mapped to the problem of density matrix evolution in terms of the Lindblad equation [29,30]. If the Hamiltonian of the system is quadratic and the Lindblad operators are linear, the solution of Lindblad equation can be reduced to solving quadratic nonHermitian Liouvillian matrix [31,32]. While topological edge states of non-Hermitian Hamiltonians have been intensively studied in recent years [33-40], it is insufficient to study the unique features associated with non-Hermitian topology in open quantum systems [41-48].

One of unique features of non-Hermitian systems is the non-Hermitian skin effect [13], which is characterized by the emergence of the majority of eigenstates accumulated at one of the boundaries with a remarkably different eigenvalue spectral under the open versus periodic boundary conditions, and breakdown of conventional bulk boundary correspondence [12-14,49-61]. Both phenomena can be understood in the scheme of non-Bloch band theory by introducing the concept of the generalized Brillouin zone (GBZ), which is composed

\footnotetext{
*schen@iphy.ac.cn

Published by the American Physical Society under the terms of the Creative Commons Attribution 4.0 International license. Further distribution of this work must maintain attribution to the author(s) and the published article's title, journal citation, and DOI.
}

of all possible values of the complex analytical continuation of Bloch momentum $k$ determined by the GBZ equation [62-65]. For systems with different symmetries, the GBZ equation may be different. Meanwhile, the skin effect is also unveiled to be originated from intrinsic non-Hermitian topology, which can be enriched by symmetry. This leads to the discovery of $\mathbb{Z}$ and $\mathbb{Z}_{2}$ non-Hermitian skin effect $[63,66]$.

Very recently, critical skin effect (CSE) was dubbed to describe a novel critical behavior in the non-Hermitian system with the energy spectrum and wave function jumping discontinuously across a critical point [67]. It is found that CSE occurs whenever two one-band subsystems with different GBZs are coupled by even a vanishingly small $k$ independent perturbation, but does not occur for subsystems with the same GBZ [67].

Both the skin effect and CSE can be viewed as some kinds of instabilities of spectra of non-Hermitian Hamiltonians, which are sensitive to boundary conditions. A natural question arises: Does the boundary-dependent instability of spectra of Liouvillians or damping matrices induce some novel phenomena in quantum open systems?

For an open quantum system with its damping matrix exhibiting the $\mathbb{Z}$-type skin effect, the chiral damping has been uncovered [47]. In this work, we shall explore new physical phenomenon associated with the $\mathbb{Z}_{2}$ skin effect and CSE in open quantum systems. In contrast to the previous work [47], we consider quantum open systems with internal spin degree, in which the time-reversal symmetry may exist and plays an important role. We demonstrate the existence of helical damping related to $\mathbb{Z}_{2}$ skin effect. The helical damping is characterized by the evolution of relative particle number $\tilde{n}(x, t)$ with exponentially decreasing intervals and power-law-decreasing intervals distinguished by sharp wave fronts with opposite propagation directions. When the 
coupling perturbation breaks the time-reversal symmetry, we demonstrate that the corresponding damping matrix exhibits CSE which leads to the disappearance of helical damping under the thermodynamic limit. Furthermore, we find that the coupled subsystems with same GBZs can exhibit CSE, for which we call it anomalous critical skin effect, and also give an example that coupled subsystems with different GBZs do not support CSE. We shall explain these phenomena and demonstrate that the change of the GBZ equation is the necessary condition of CSE, as long as the non-Bloch band theory works. Our research provides a framework for studying CSE and symmetry protected skin effect in open quantum systems and reveals the origin of CSE.

\section{HELICAL DAMPING}

Open Markovian quantum systems satisfy the Lindblad master equation $[29,30]$ :

$$
\frac{d \rho}{d t}=-i[H, \rho]+\sum_{\mu}\left(2 L_{\mu} \rho L_{\mu}^{\dagger}-\left\{L_{\mu}^{\dagger} L_{\mu}, \rho\right\}\right),
$$

where $\rho$ is the density matrix, $H$ is the Hamiltonian, and $L_{\mu}$ are Lindblad operators describing quantum jumps induced by the coupling to the environment. Consider a one-dimensional (1D) lattice with the unit cell composed of two orbits (sublattices) and each site can be occupied by spin-up and spin-down fermions. In the momentum space, the Hamiltonian is given by

$$
h(k)=t_{1} \sigma_{x}+\left(t_{2} \sigma_{y}+\delta_{1} \tau_{x}\right) \sin k+t_{2} \sigma_{x} \cos k+\delta_{2} \sigma_{z} \tau_{x},
$$

where $\sigma_{x, y, z}$ and $\tau_{x, y, z}$ act on orbit and spin degrees of freedom, respectively. Here we consider quantum jump processes described by the following Lindblad operators:

$$
\begin{array}{ll}
L_{x \uparrow}^{l}=\sqrt{\frac{\gamma_{l}}{2}}\left(c_{x A \uparrow}-i c_{x B \uparrow}\right), \quad L_{x \uparrow}^{g}=\sqrt{\frac{\gamma_{g}}{2}}\left(c_{x A \uparrow}^{\dagger}+i c_{x B \uparrow}^{\dagger}\right), \\
L_{x \downarrow}^{l}=\sqrt{\frac{\gamma_{l}}{2}}\left(c_{x A \downarrow}+i c_{x B \downarrow}\right), \quad L_{x \downarrow}^{g}=\sqrt{\frac{\gamma_{g}}{2}}\left(c_{x A \downarrow}^{\dagger}-i c_{x B \downarrow}^{\dagger}\right),
\end{array}
$$

where $s=\uparrow, \downarrow, x$ is the cell index, and $o=A, B$ refer to the spin and orbit index, respectively.

Define $\Delta_{m, n}=\operatorname{Tr}\left(c_{m}^{\dagger} c_{n} \rho\right)$ with $m, n=(x, s, o)$, and $\tilde{\Delta}=$ $\Delta-\Delta_{s}$ with $\Delta_{s}$ denoting the steady value of $\Delta$. After some derivations (see Appendix A for details), the dynamical evolution of $\tilde{\Delta}$ is governed by

$$
\frac{d \tilde{\Delta}(t)}{d t}=X \tilde{\Delta}(t)+\tilde{\Delta}(t) X^{\dagger},
$$

which gives rise to $\tilde{\Delta}(t)=e^{X t} \tilde{\Delta}(0) e^{X^{\dagger} t}$ with the damping matrix in the momentum space given by

$$
\begin{aligned}
X= & i\left[\begin{array}{cc}
H_{\mathrm{nSSH}}(k)+\frac{i \gamma}{2} & \delta_{1} \sin k+\delta_{2} \sigma_{z} \\
\delta_{1} \sin k+\delta_{2} \sigma_{z} & H_{\mathrm{nSSH}}^{T}(-k)+\frac{i \gamma}{2}
\end{array}\right] \\
= & \left(-\frac{\gamma}{2}+i t_{1} \sigma_{x}+\frac{\gamma}{2} \sigma_{y} \tau_{z}\right)+i\left(t_{2} \sigma_{y}+\delta_{1} \tau_{x}\right) \sin k \\
& +i t_{2} \sigma_{x} \cos k+i \delta_{2} \sigma_{z} \tau_{x},
\end{aligned}
$$

where $\Delta_{s}=\frac{\gamma_{g}}{\gamma} \mathbb{I}$ with $\gamma=\gamma_{l}+\gamma_{g}$ and

$$
H_{\mathrm{nSSH}}(k)=\left(t_{1}+t_{2} \cos k\right) \sigma_{x}+\left(t_{2} \sin k-\frac{i \gamma}{2}\right) \sigma_{y}
$$
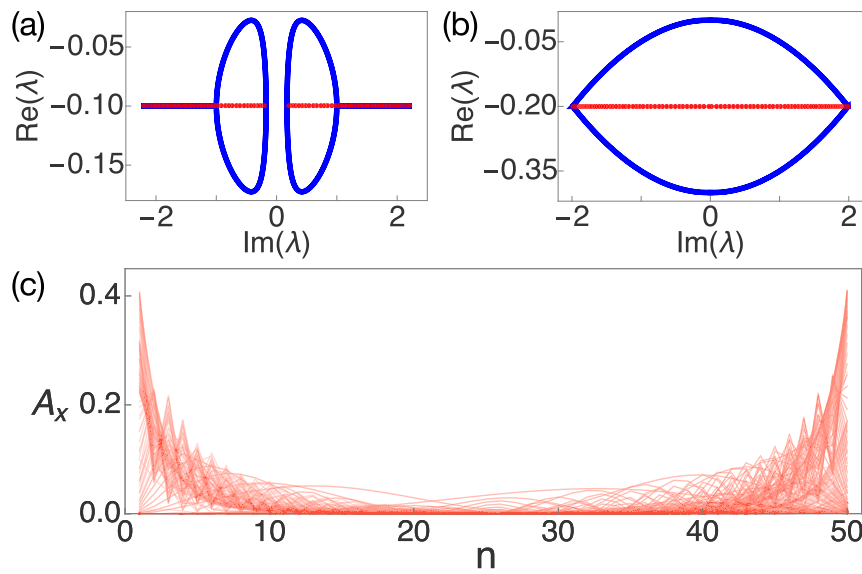

FIG. 1. (a) and (b) Red (blue) dots denote open (periodic) boundary spectrum of $X$. The parameter values are $t_{1}=1.2, t_{2}=1, \gamma=$ $0.2, \delta_{1}=0.1, \delta_{2}=0$ for (a) and $t_{1}=t_{2}=1, \gamma=0.4, \delta_{1}=0.1, \delta_{2}=$ 0 for (b). (c) Sum of modular squares of the wave function in each unit cell $A_{x}$ for the open boundary damping matrix with the same parameters as in (b).

takes the same form of the non-Hermitian Su-SchriefferHeeger (SSH) model $[4,9,13]$.

When $\delta_{2}=0, X$ has time-reversal symmetry, as it fulfills

$$
C X(-k)^{T}=X(k) C,
$$

with $C=i \tau_{y}$ [19]. It should be noticed that the symmetry is different from time-reversal symmetry in the Hermitian case $\left(C X(-k)^{*}=X(k) C\right)$, since $H^{T} \neq H^{*}$ in the non-Hermitian system. Such a symmetry belongs to one of 54 classes of non-Hermitian line gap symmetries, and cannot be described by 38 non-Hermitian symmetries of the point gap. For example, $q H^{*} q^{\dagger}=H$ and $q H^{*} q^{\dagger}=-H$ belong to the same class according to the 38 -fold way, but have different real line gap classification. The gap discussed in our article is a real line gap, which should be described by the 54-fold way [19].

We can get the eigenvalues of $X$ under open boundary condition (OBC) and periodic boundary condition (PBC) as shown in Figs. 1(a) and 1(b). The mismatching of eigenvalues under open and periodic boundary is a characteristic sign of skin effect. Define the sum of modular squares of $X$ 's eigen-wave-function in each unit cell as $A_{x}=\sum_{o, s}\left|\left(\Psi_{x, o}^{s}\right)\right|^{2}$. In Fig. 1(c), we show the distribution of $A_{x}$ under the OBC. The majority of eigenstates of $X$ are found to localize on left and right boundaries, which gives the signature for the existence of skin effect. Putting two identical models together and adding a small symmetry-allowed perturbation, we find lots of skin modes disappeared (see Fig. 6, for example). This has been viewed as an additional evidence of the $\mathbb{Z}_{2}$ skin effect.

Given $n_{x, s, o} \equiv \Delta_{(x, s, o),(x, s, o)}$ denoting the particle number with spin $s$ and orbit $o$ at site $x$, we define the local damping as $D_{x}(t)=\sqrt{\sum_{s, o}\left(\frac{d n_{x, s, o}(t)}{d t}\right)^{2}}$ and relative local particle number $\tilde{n}_{x}(t)=\sum_{s, o} \tilde{\Delta}_{(x, s, o),(x, s, o)}$. In Figs. 2(a) and 2(b) we display $\log \left(D_{x}(t)\right)$ as a function of $t$ for different $x$. While $D_{x}(t)$ under PBC is always a power-law function of $t, D_{x}(t)$ under OBC changes from a power-law function to an exponential function of $t$ during the evolution. We find that the transition time $t_{c}$ 

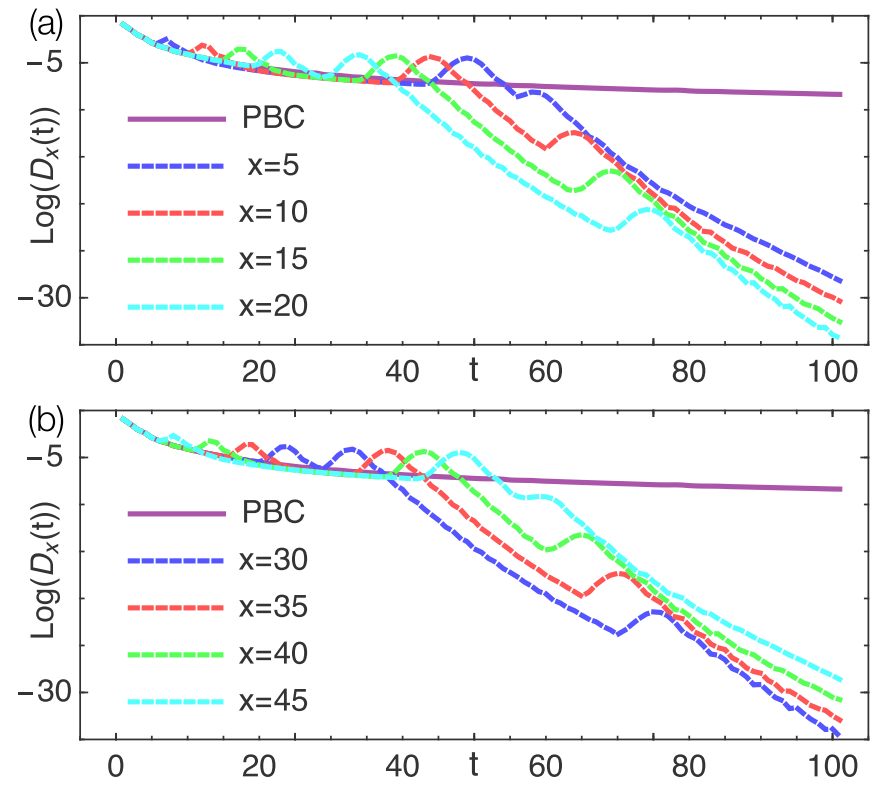

FIG. 2. (a) and (b) Illustration of site-resolved relative local particle number damping of the open boundary chain (dashed lines) and periodic chain (solid curve). The parameters are set as $t_{1}=t_{2}=1$, $\gamma=0.4, \delta_{1}=0.1$, and $\delta_{2}=0$.

decreases as $x$ increases for $0<x<20$, and increases as $x$ increases for $30<x<50$. In order to see more clearly the relation between $t_{c}$ and $x$, we plot the relative local particle number evolution in Figs. 3(a) and 3(b) for the periodic and open boundary system, respectively. There are three main colors in the figures: dark blue, blue, and purple, which are
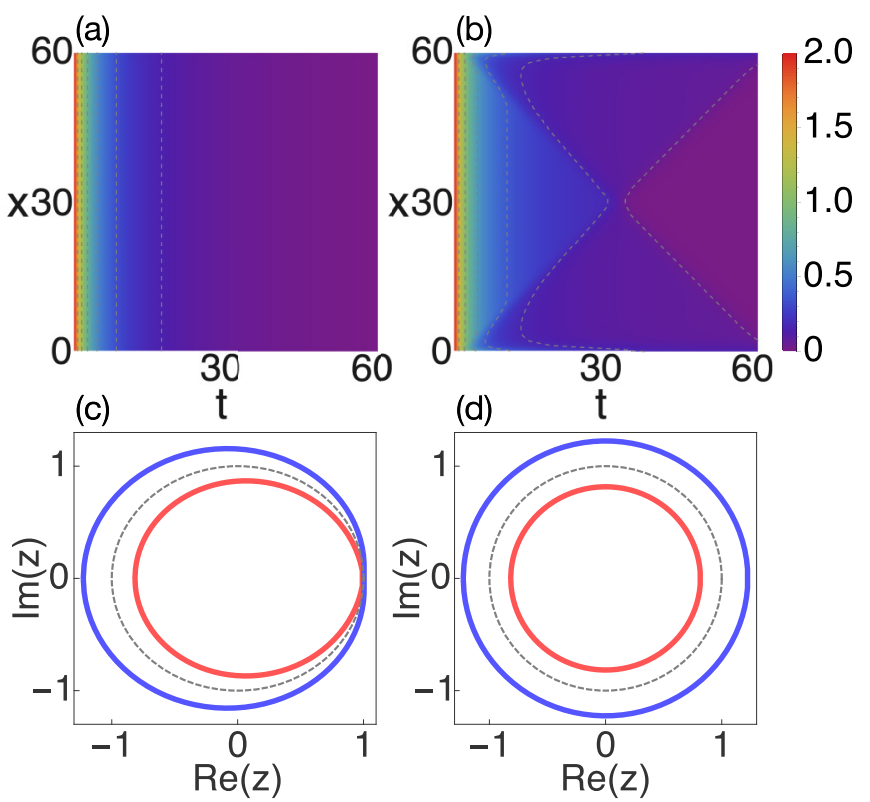

FIG. 3. (a) and (b) The time evolution of $\tilde{n}_{x}(t)$ for the system under PBC and OBC, respectively. (c) and (d) The GBZ of damping matrix $X$, whereas the solid line shows GBZ and the dotted line shows the BZ, which is a unit circle given by the trajectory of $|z|=1$. The parameters are $t_{1}=t_{2}=1, \gamma=0.4, \delta_{1}=0.1, \delta_{2}=0$ for (a), (b), and (c), and $t_{1}=t_{2}=1, \gamma=0.4, \delta_{1}=\delta_{2}=0$ for (d). separated by two straight lines as shown in Fig. 3(b). The separatrix of the dark blue area and purple area is the transition line. Such a phenomena is dubbed as helical damping. Nevertheless, the $\mathbb{Z}_{2}$ skin effect is not the sufficient condition of helical damping (see Appendix B), and we also require the Liouvillian gap of periodic lattice to be zero and the open boundary Liouvillian gap to be nonzero, where the Liouvillian gap is defined as

$$
\Lambda_{g}=\min \left[2 \operatorname{Re}\left(-\lambda_{n}\right)\right]
$$

with $\lambda_{n}$ the eigenvalues of $X$. We notice that $\frac{\left[\tilde{n}_{x}(t)\right]_{\mathrm{OBC}}}{\left[\tilde{n}_{x}(t)\right]_{\mathrm{PBC}}}$ may exhibit helical behavior even if the periodic boundary system is gapped (see Appendix B). When the periodic boundary system is gapped (gapless), the short-time behavior of damping fulfills exponential (power) law for both the periodic and open boundary systems, since it costs time for sites located not on the boundary to get the boundary information. On the other hand, long-time behavior of OBC's damping fulfills exponential (power) law when the open boundary system is gapped (gapless), which will be explained further below.

Now we use non-Bloch band theory to explain helical damping. For open boundary system the bulk wave function and eigenvalue of the $X$ matrix can be obtained by replacing $X(k)$ with $X(k+i \kappa)$. All possible values of $z=e^{i(k+i \kappa)}$ constitute GBZ. The complex number $z$ can be derived from the characteristic equation,

$$
f(z, \lambda) \equiv \operatorname{det}(X(z)-\lambda)=0 .
$$

The GBZ of the damping matrix can be determined by requiring a pair of zeros of the polynomial $f(z, \lambda)$ to fulfill GBZ equation,

$$
\left|z_{\mu}\right|=\left|z_{\nu}\right|
$$

for the same $\lambda$ and certain $\mu, v$. In Figs. 3(c) and 3(d), we display the GBZ of the system with different parameters, which is composed of two closed curves with one inside and one outside the Brillouin zone (BZ). The relative local particle number can be decomposed into each of GBZ modes: $\tilde{n}_{k+i \kappa}=\sum_{s, o}\left\langle x, s, o\left|e^{X(k+i \kappa) t} e^{X^{\dagger}(k+i \kappa) t}\right| x, s, o\right\rangle$. It follows $\max \left\{\tilde{n}_{k+i \kappa}\right\} \propto e^{2 \kappa\left(x_{1}-x\right)-\gamma t}$ (see Appendix C). Define the velocity for the open boundary system as $v_{k+i \kappa, \alpha}=$ $\operatorname{Re}\left(\frac{i \partial \lambda_{k+i \kappa, \alpha}}{\partial k}\right)$, where $\alpha$ is the band index, and $\lambda_{k+i \kappa, \alpha}$ is the eigenvalue of $X(k+i \kappa)$ corresponding to the $\alpha$ band. For simplicity we use $v$ to label $v_{k+i \kappa}$ in the following text. If the parameter settings are the same as in Fig. 3(b), we can get $v_{\max }=-v_{\min } \approx 1$ and $\kappa_{\max }=-\kappa_{\min }=0.2$ at $k=$ $\pi$. For $v=1, \kappa=-0.2$ and $x_{1}-x=-v t, x=x_{1}+v t \geqslant t$, and $e^{2 \kappa\left(x_{1}-x\right)-\gamma t}=e^{(-2 v \kappa-\gamma) t}=1$. The decay factor cancels out, and the particle number damping fulfills a power law. Similarly, for $v=-1, \kappa=0.2$, and $x=x_{1}+v t \leqslant L-t$, the decay factor also cancels out. For $x=x_{1}+v t<t$ and $x=x_{1}+v t>L-t$, this factor cannot be canceled out, and the relative particle number damping obeys an exponential law. Due to the existence of time-reversal symmetry, we have $n_{x}(t)=n_{L-x}(t)$, which distributes symmetrically about $x=\frac{L}{2}$. 

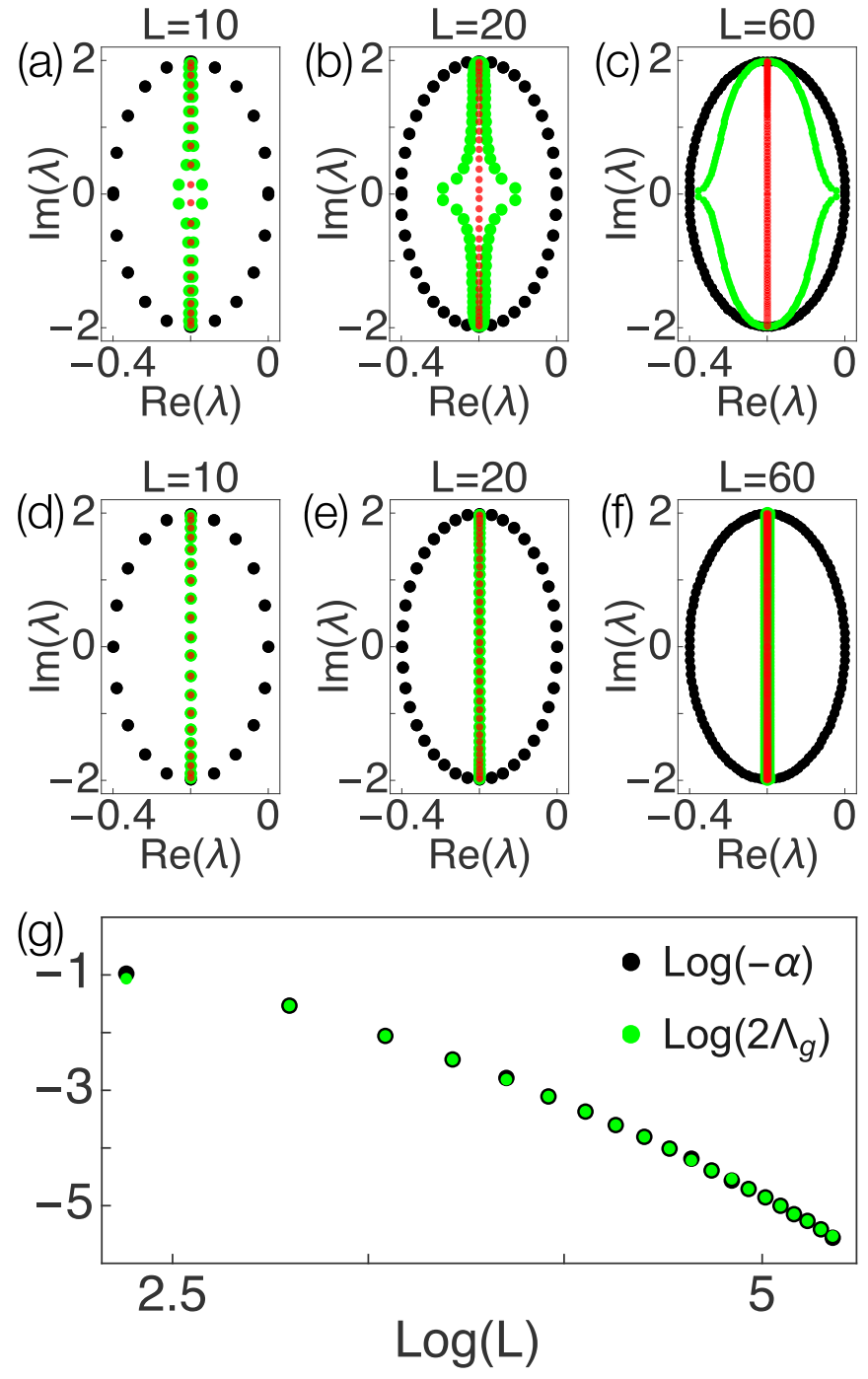

FIG. 4. Open (green) and periodic (black) boundary spectrum of $X$ for systems with $t_{1}=t_{2}=1, \gamma=0.4$ and other parameters set as $\delta_{1}=0, \delta_{2}=0.02$ for (a), (b), and (c) with different sizes, and $\delta_{1}=$ $0.02, \delta_{2}=0$ for (d), (e), and (f) with different sizes, respectively. The red dots denote the open boundary spectrum of $X$ with parameters set as $t_{1}=t_{2}=1, \gamma=0.4, \delta_{1}=\delta_{2}=0$. (g) Long-time damping slopes $\alpha$ and $-2 \Lambda_{g}$ as a function of size $L$ with parameters set as $t_{1}=t_{2}=$ $1, \gamma=0.4, \delta_{1}=0, \delta_{2}=0.02$.

\section{DYNAMICAL CRITICAL SKIN EFFECT}

When the system exhibits CSE, the open boundary energy spectrum is not continuous under the small change of parameters in the thermodynamic limit. For the finite size system, the open boundary spectrum is always continuous under the small change of parameters. Therefore, if CSE occurs, the eigenvalue spectrum of the system varies greatly with the size of the system. Here we study whether the perturbation $\delta_{1}$ or $\delta_{2}$ will cause CSE, which is possible to be detected in dynamical experiments. With parameters set as $\delta_{1}=0.02$, $\delta_{2}=0$ or $\delta_{1}=0, \delta_{2}=0.02$, we calculate the spectrum of $X$ for systems with different sizes. The result is shown in Fig. 4. In Figs. 4(a)-4(c), we display the spectrum of damping matrix with $\delta_{1}=0$ and $\delta_{2}=0.02$ for different system sizes. While
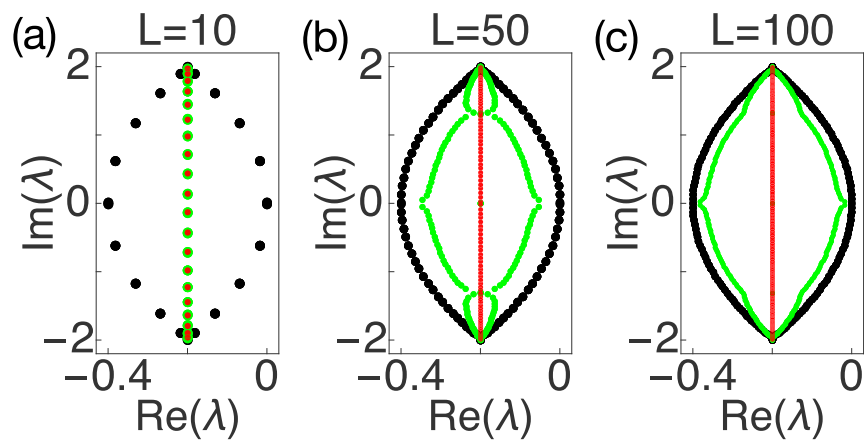

FIG. 5. Open (green) and periodic (black) boundary spectrum with parameters $t_{1}=t_{2}=1, \gamma=0.4, \delta_{1}=0.1, \delta_{2}=0, \delta_{3}=$ 0.01 for (a), (b), and (c) with different sizes $L=10,50$, and 100, respectively. The red dots denote the open boundary spectrum of $X_{1}$ with parameters $t_{1}=t_{2}=1, \gamma=0.4, \delta_{1}=0.1, \delta_{2}=\delta_{3}=0$.

the periodic spectrum is not sensitive to the system size $L$, the obvious change of open boundary spectrum with the increase of $L$ indicates the existence of dynamical CSE, and the open boundary Liouvillian gap $\Lambda_{g}$ decreases with the increase of the system size.

To measure the Liouvillian gap from dynamical experiment, we derive the relationship between relative particle number evolution and the Liouvillian gap. The relative particle number is $\tilde{n}_{x}(t)=$ $\sum_{i, j, s, o} e^{\left(\lambda_{i}+\lambda_{j}^{*}\right) t}\left\langle x, s, o \mid \Psi_{i}\right\rangle_{R L}\left\langle\Psi_{i} \mid \Psi_{j}\right\rangle_{R L}\left\langle\Psi_{j} \mid x, s, o\right\rangle$, where subscripts $R$ and $L$ denote the right and left eigenvectors of $X$. Consider the case with large enough $t$, for which modes with $-\operatorname{Re}\left(\lambda_{i}+\lambda_{j}^{*}\right)>2 \Lambda_{g}$ can be omitted, and it follows $\tilde{n}_{x}(t) \approx c e^{-2 \Lambda_{g} t}$. Assume that $\log \left(\tilde{n}_{x}(t)\right)=\alpha(t) t+\beta(t)$, then $\alpha \approx-2 \Lambda_{g}$. We numerically calculate the values of $2 \Lambda_{g}$ and $\alpha$ for different size systems. As illustrated in Fig. 4(g), the numerical results are consistent with our theoretical analysis. We also analyze the scaling of the Liouvillian gap with the system size, which indicates $\log \left(2 \Lambda_{g}\right) \approx-2.3 \log (L)+6.8$ around $L=200$ and the absolute value of this slope increases as $L$ increases. When the system is large enough so that $-2 \Lambda_{g}>\alpha_{\max }$, the helical damping is hidden. On the other

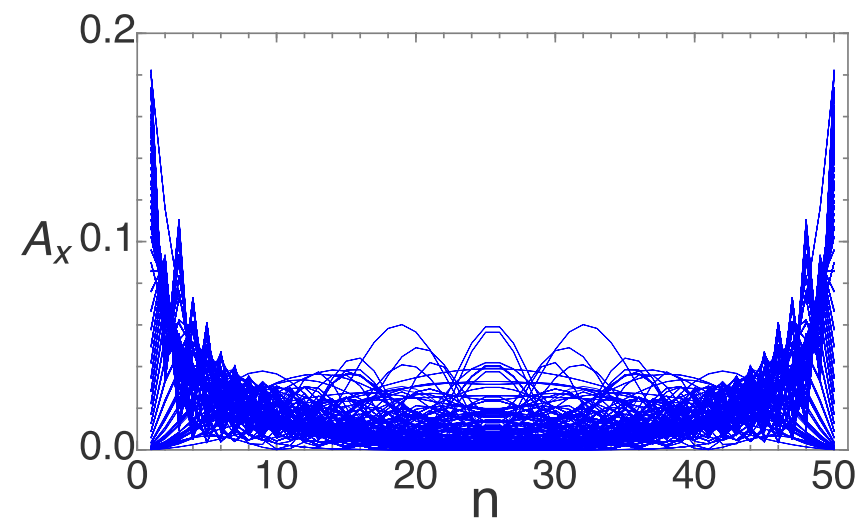

FIG. 6. Sum of modular squares of the wave function in each unit cell $A_{x}$ for the open boundary damping matrix of $X_{1}$. The parameters of $X_{1}$ are set as $t_{1}=t_{2}=1, \gamma=0.4, \delta_{1}=0.1, \delta_{2}=0, \delta_{3}=0.01$. The system size is $L=50$. 


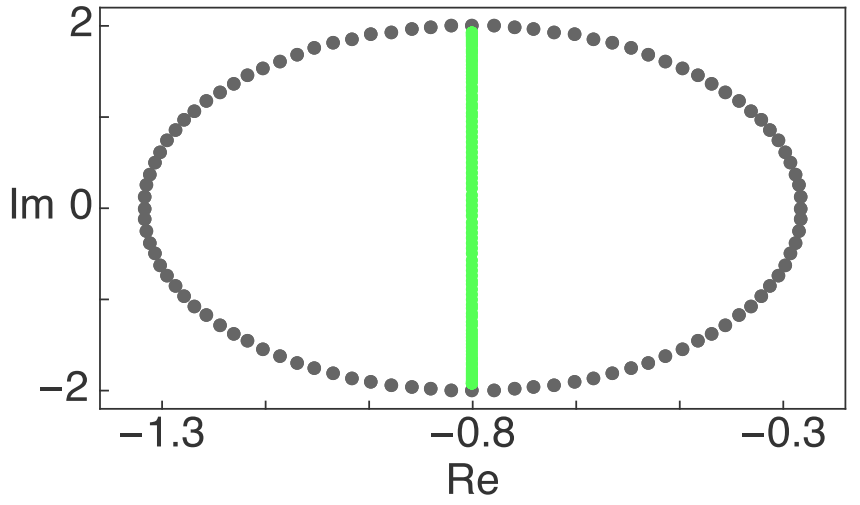

FIG. 7. Open (green) and periodic (black) boundary spectra of the $X$ matrix. The parameters are taken as $t_{1}=1, \gamma_{g}=0.4, \delta=0.3$ with the system size $L=100$.

hand, when the system is small enough so that $-2 \Lambda_{g}<\alpha_{\min }$, the helical damping is manifested. Here $\alpha_{\max } / \alpha_{\min }$ is the maximum/minimum slope of $\left[\log \left(\tilde{n}_{x}(t)\right)\right]_{\mathrm{PBC}}$ in the

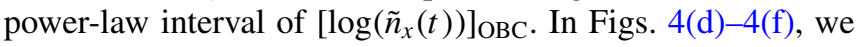
set $\delta_{1}=0.02$ and $\delta_{2}=0$. It is clearly shown that there is no CSE, and the open boundary Liouvillian gap $\Lambda_{g}(L)$ does not change as the system size increases. Therefore we can detect the presence of CSE by measuring the damping spectra of systems with different sizes. Remarkably, we construct an example that the system is coupled by two irreducible subsystems with different GBZs but does not exhibit CSE. Specifically, $X$ is constructed by coupling two systems $i H_{\mathrm{nSSH}}(k)-\frac{\gamma}{2}$ and $i H_{\mathrm{nSSH}}^{T}(-k)-\frac{\gamma}{2}$, which have different GBZs.

Here we give an explanation in terms of GBZ. The bulk spectrum of open boundary $X$ is composed of eigenvalues of $X(k+i \kappa)$, and thus it is a continuous function of GBZ. On the other hand, the solution of a certain GBZ equation is a
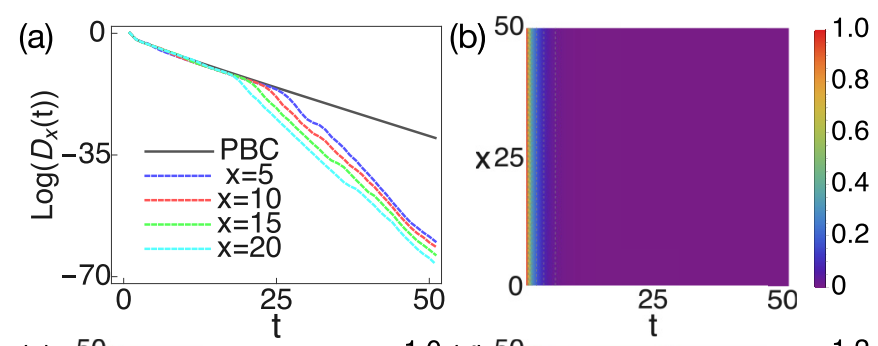

(c)
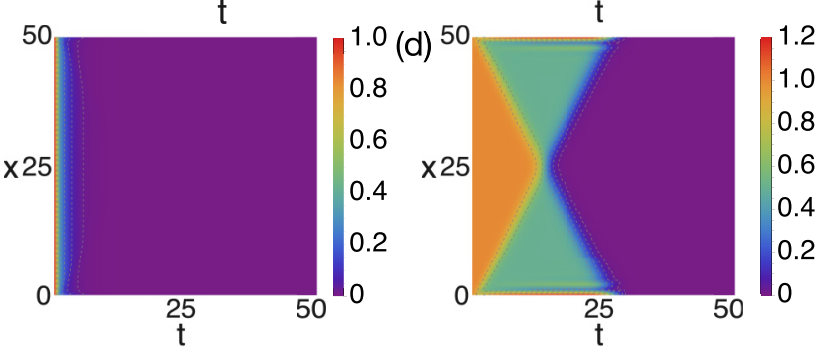

FIG. 8. (a) Illustration of the site-resolved relative local particle number damping for different cells. (b) and (c) The evolution of $\left|\tilde{n}_{x}(t)\right|$ for system under (b) PBC and (c) OBC, respectively. (d) The evolution of $\frac{\left(\left|\tilde{n}_{x}(t)\right|\right)_{\mathrm{OBC}}}{\left(\left|\tilde{n}_{x}(t)\right|\right)_{\mathrm{PBC}}}$. The parameters are set as $t_{1}=1, \gamma_{g}=$ $0.4, \delta=0.3$ with the system size $L=50$. continuous function of parameters of $X$. Therefore the only origin of discontinuity of energy spectrum comes from the change of the GBZ equation. For case 1 with $\delta_{1}=\delta_{2}=0, X$ is reducible, and the characteristic polynomial is $f_{1}(z, \lambda)=$ $\operatorname{det}\left(i H_{\mathrm{nSSH}}-\frac{\gamma}{2}-\lambda I\right)$ and $f_{2}(z, \lambda)=\operatorname{det}\left(i H_{\mathrm{nSSH}}^{T}-\frac{\gamma}{2}-\lambda I\right)$, where $z=e^{i k}$. The roots of $f_{1}=0$ and $f_{2}=0$ are $z_{1}^{a}, z_{2}^{a}$ and $z_{1}^{b}, z_{2}^{b}$, respectively, where $\left|z_{1}^{a}\right| \leqslant\left|z_{2}^{a}\right|, z_{i}^{b}=1 / z_{i}^{a}(i=1,2)$. The GBZ equations are $\left|z_{1}^{a}\right|=\left|z_{2}^{a}\right|$ and $\left|z_{1}^{b}\right|=\left|z_{2}^{b}\right|[62,63]$. If $X$ is an irreducible non-Hermitian matrix, the characteristic polynomial is $f(z, \lambda)=\operatorname{det}(X-\lambda I)$, and the solution of $f(z, \lambda)=0$ is $z_{1}, z_{2}, z_{3}, z_{4}$, where $\left|z_{1}\right| \leqslant\left|z_{2}\right| \leqslant\left|z_{3}\right| \leqslant\left|z_{4}\right|$. For case 2 with $\delta_{1}=0$ and $\delta_{2} \neq 0, X$ does not have any symmetry, and the GBZ equation is $\left|z_{2}\right|=\left|z_{3}\right|$. For case 3 with $\delta_{1} \neq 0$ and $\delta_{2}=0, X$ has time-reversal symmetry. The GBZ equations are $\left|z_{1}\right|=\left|z_{2}\right|$ and $\left|z_{3}\right|=\left|z_{4}\right|$ and the roots satisfy $z_{2+i}=1 / z_{3-i}(i=1,2)$ [65]. We emphasize that the GBZ equation of case 1 and case 3 are equivalent (see Appendix D). So there is a change of the GBZ equation from case 1 to case 2 , which causes the discontinuity of eigenvalues and wave functions under the time-reversal-breaking perturbation. The GBZ equation does not change from case 1 to case 3 , therefore no CSE occurs in this process.

Next we construct a model which is composed of two coupled irreducible subsystems with the same GBZs but shows CSE, i.e., exhibiting the anomalous critical skin effect. We consider the model described by

$$
X_{1}=\left[\begin{array}{cc}
X & \delta_{3} \sigma_{x} \tau_{y} \cos k \\
-\delta_{3} \sigma_{x} \tau_{y} \cos k & X
\end{array}\right] .
$$

The parameters are set as $t_{1}=t_{2}=1, \gamma=0.4, \delta_{1}=$ $0.1, \delta_{2}=0$, and $\delta_{3}=0.01$. We note that the coupling term of $\delta_{3}$ does not break the time-reversal symmetry. Although the damping matrix $X_{1}$ is constructed by coupling two identical systems of $X$ described by Eq. (5), next we shall demonstrate that it exhibits CSE. In Fig. 5, we display the open boundary and periodic boundary spectrum of $X_{1}$. While the periodic boundary spectrum is not sensitive to the lattice size, the shape of the open boundary spectrum changes obviously with the increase in the lattice size. Such an obvious change of the open boundary spectrum is induced by the symmetry-allowed perturbation term of $\delta_{3}$. It is shown that the doubled $\mathbb{Z}_{2}$ skin effect is not stable to symmetry-allowed perturbation and anomalous CSE emerges.

In Fig. 6, we show the distribution of $A_{x}$ under the OBC, where $A_{x}=\sum_{\alpha}\left|\Psi_{x \alpha}\right|^{2}$ is the sum of modular squares of the wave function amplitude of $X_{1}$ in each unit cell, $\alpha$ is degrees of freedom in each cell, and $x$ is the cell index. It is clear that some eigenstates of $X_{1}$ spread over all the lattices. While many skin modes disappear, there are still some skin modes, as the open boundary spectrum is not perfectly coincident with the spectrum under PBC. Our results suggest that the emergence of critical skin effect, instead of disappearance of skin modes, for two identical nontrivial $\mathbb{Z}_{2}$ systems coupled with symmetry-allowed perturbation is more suitable to serve as an evidence for the $\mathbb{Z}_{2}$ skin effect.

When $\delta_{3}=0$, the GBZ equations of $X_{1}$ are $\left|z_{1}\right|=\left|z_{2}\right|$ and $\left|z_{3}\right|=\left|z_{4}\right|$. When $\delta_{3} \neq 0$, the characteristic polynomial of $X_{1}$ is $g(z, \lambda)=\operatorname{det}\left(X_{1}(z)-\lambda I\right)$, with the solution of $g(z, \lambda)=0$ given by $\tilde{z}_{1}, \tilde{z}_{2}, \ldots, \tilde{z}_{8}$, where $\left|\tilde{z}_{1}\right| \leqslant\left|\tilde{z}_{2}\right| \leqslant \ldots \leqslant\left|\tilde{z}_{8}\right|$. The GBZ 

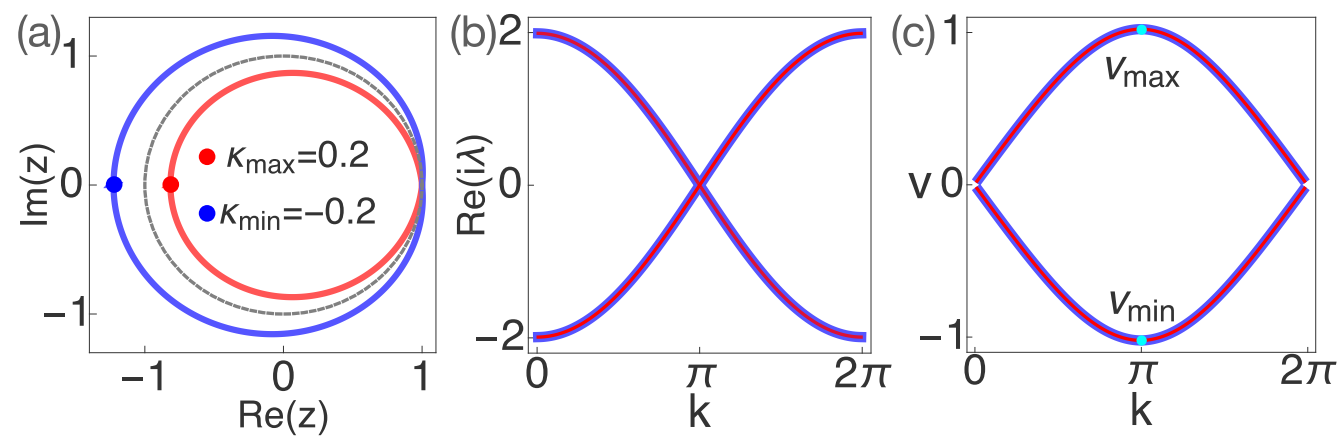

FIG. 9. (a) The GBZ of Eq. (5). Parameter values are $t_{1}=t_{2}=1, \gamma=0.4, \delta_{1}=0.1$, and $\delta_{2}=0$. Two bands correspond to red curves and the other two to blue curves. (b) $\operatorname{Re}\left(i \lambda_{k+i k}\right)$ as a function of $k$. There are four bands with each band corresponding to one curve, among which there are two red curves and two blue curves. (c) Velocity $v=\operatorname{Re}\left(\frac{i \partial \lambda_{k+i k, \alpha}}{\partial k}\right)$ as a function of $k$, and $\alpha$ is the band index. Curves in (a)-(c) with the same color are corresponding to each other.

equations are $\left|\tilde{z}_{3}\right|=\left|\tilde{z}_{4}\right|$ and $\left|\tilde{z}_{5}\right|=\left|\tilde{z}_{6}\right|$. The GBZ equations also change when CSE occurs. The reason for the discontinuity is the change in GBZ equations. Furthermore, changes in GBZ equations require changes of symmetries of the system or number of roots of the characteristic equation. In this case, when $\delta_{3}$ changes from zero to nonzero, the symmetry $\tilde{\sigma}_{z} X_{1}=X_{1} \tilde{\sigma}_{z}$ disappears, where $\tilde{\sigma}_{z}$ is a Pauli matrix acting on the doubled space (not spin or orbits). Therefore, changes in the GBZ equation are necessary conditions for CSE, but the reverse is not correct. An example is that no matter how the GBZ equation changes for a Hermitian system, there is no CSE. The Hermitian condition $H^{\dagger}=H$ keeps the GBZ to be always a unit circle, even if the GBZ equation changes.

\section{SUMMARY AND DISCUSSION}

In summary, we have unveiled the occurrence of helical damping in the open quantum system with $\mathbb{Z}_{2}$ skin effect if the open boundary spectrum of the damping matrix is gapped and the periodic boundary spectrum is gapless. When the time-reversal symmetry is broken by perturbations, we demonstrated the existence of dynamical CSE, which is characterized by the vanishing of helical damping in the thermodynamic limit although it still exists in the small size system. Coupling two identical models with $\mathbb{Z}_{2}$ skin effects by perturbation, we can realize anomalous critical skin effect. Based on non-Bloch band theory, we also unveiled the discontinuity in CSE coming from the change of the GBZ equation.

Finally, we remark that the possible physical realization of the helical damping can be explored in an equivalent model.
Through a unitary transformation $\left(\sigma_{x} \rightarrow \sigma_{x}, \sigma_{y} \rightarrow \sigma_{z}, \sigma_{z} \rightarrow\right.$ $\sigma_{y}$ ), the open system described by the Lindblad equation with the Hamiltonian and Lindblad operators given by Eqs. (2) and (3) is transformed to a corresponding Lindblad equation with the Hamiltonian in momentum space given by

$$
h(k)=t_{1} \sigma_{x}+\left(t_{2} \sigma_{z}+\delta_{1} \tau_{x}\right) \sin k+t_{2} \sigma_{x} \cos k+\delta_{2} \sigma_{y} \tau_{x},
$$

and Lindblad operators given by

$$
\begin{aligned}
& L_{x \uparrow}^{l}=\sqrt{\gamma_{l}} c_{x A \uparrow}, \quad L_{x \uparrow}^{g}=\sqrt{\gamma_{g}} c_{x A \uparrow}^{\dagger}, \\
& L_{x \downarrow}^{l}=\sqrt{\gamma_{l}} c_{x B \downarrow}, \quad L_{x \downarrow}^{g}=\sqrt{\gamma_{g}} c_{x B \downarrow}^{\dagger} .
\end{aligned}
$$

Such a model can be realized by introducing on-site singleparticle gain and loss, which might be easier to be manipulated than the Lindblad operators described by Eq. (3) in realistic physical systems, such as artificial photonic systems and topolectrical circuits $[68,69]$. The helical damping is also expected to be observed in this system.

\section{ACKNOWLEDGMENTS}

The work is supported by NSFC under Grant No. 11974413, the National Key Research and Development Program of China (Grants No. 2016YFA0300600 and No. 2016YFA0302104), and the Strategic Priority Research Program of Chinese Academy of Sciences under Grant No. XDB33000000.

\section{APPENDIX A: DERIVATION OF EVOLUTION EQUATION OF SINGLE-PARTICLE CORRELATION MATRIX}

Here we derive the expression of the evolution equation of single-particle correlation matrix $\Delta$ with $\Delta_{m n}=\operatorname{Tr}\left(c_{m}^{\dagger} c_{n} \rho\right)$. Inserting the Lindbald equation into

we have

$$
\left(\frac{d \Delta_{m n}}{d t}\right)=\operatorname{Tr}\left(c_{m}^{\dagger} c_{n} \frac{d \rho}{d t}\right),
$$

$$
\begin{aligned}
\left(\frac{d \Delta_{m n}}{d t}\right) & =\operatorname{Tr}\left[c_{m}^{\dagger} c_{n}\left(-i[H, \rho]+\sum_{\mu}\left(2 L_{\mu} \rho L_{\mu}^{\dagger}-\left\{L_{\mu}^{\dagger} L_{\mu}, \rho\right\}\right)\right)\right] \\
& =\operatorname{Tr}\left[-i c_{m}^{\dagger} c_{n} H \rho+i c_{m}^{\dagger} c_{n} \rho H+\sum_{\mu}\left(2 c_{m}^{\dagger} c_{n} L_{\mu} \rho L_{\mu}^{\dagger}-c_{m}^{\dagger} c_{n} L_{\mu}^{\dagger} L_{\mu} \rho-c_{m}^{\dagger} c_{n} \rho L_{\mu}^{\dagger} L_{\mu}\right)\right]
\end{aligned}
$$




$$
\begin{aligned}
& =\operatorname{Tr}\left\{\left[-i c_{m}^{\dagger} c_{n} H+i H c_{m}^{\dagger} c_{n}+\sum_{\mu}\left(2 L_{\mu}^{\dagger} c_{m}^{\dagger} c_{n} L_{\mu}-c_{m}^{\dagger} c_{n} L_{\mu}^{\dagger} L_{\mu}-L_{\mu}^{\dagger} L_{\mu} c_{m}^{\dagger} c_{n}\right)\right] \rho\right\} \\
& =\operatorname{Tr}\left\{\left[-i\left[c_{m}^{\dagger} c_{n}, H\right]+\sum_{\mu}\left(2 L_{\mu}^{\dagger} c_{m}^{\dagger} c_{n} L_{\mu}-2 L_{\mu}^{\dagger} L_{\mu} c_{m}^{\dagger} c_{n}+L_{\mu}^{\dagger} L_{\mu} c_{m}^{\dagger} c_{n}-c_{m}^{\dagger} c_{n} L_{\mu}^{\dagger} L_{\mu}\right)\right] \rho\right\} \\
& =\operatorname{Tr}\left\{\left[-i\left[c_{m}^{\dagger} c_{n}, H\right]+\sum_{\mu}\left(2 L_{\mu}^{\dagger}\left[c_{m}^{\dagger} c_{n}, L_{\mu}\right]+\left[L_{\mu}^{\dagger} L_{\mu}, c_{m}^{\dagger} c_{n}\right]\right)\right] \rho\right\} .
\end{aligned}
$$

Here $H=\sum_{j, k} h_{j k} c_{j}^{\dagger} c_{k}, L_{\mu}=L_{\mu}^{g}$ or $L_{\mu}^{l}$ with $L_{\mu}^{g}=\sum_{k} D_{\mu k}^{g} c_{k}^{\dagger}$ and $L_{\mu}^{l}=\sum_{k} D_{\mu k}^{l} c_{k}, j, k, m, n$, is the fermion index, and $\mu, \nu$ is the Lindblad operator's index. And we define $M_{j k}^{g}=\sum_{\mu} D_{\mu j}^{g *} D_{\mu k}^{g}$ and $M_{j k}^{l}=\sum_{\mu} D_{\mu j}^{l *} D_{\mu k}^{l}$. The first term gives

$$
\begin{aligned}
\operatorname{Tr}\left(-i\left[c_{m}^{\dagger} c_{n}, H\right] \rho\right) & =\sum_{j, k} \operatorname{Tr}\left(-i h_{j k}\left[c_{m}^{\dagger} c_{n}, c_{j}^{\dagger} c_{k}\right] \rho\right) \\
& =\sum_{j, k}-i h_{j k} \operatorname{Tr}\left(c_{m}^{\dagger}\left\{c_{n}, c_{j}^{\dagger}\right\} c_{k} \rho-c_{j}^{\dagger}\left\{c_{k}, c_{m}^{\dagger}\right\} c_{n} \rho\right) \\
& =\sum_{j, k}-i h_{j k} \operatorname{Tr}\left(\delta_{n, j} c_{m}^{\dagger} c_{k} \rho-\delta_{k, m} c_{j}^{\dagger} c_{n} \rho\right) \\
& =\sum_{k}-i h_{n k} \operatorname{Tr}\left(c_{m}^{\dagger} c_{k} \rho\right)+\sum_{j} i h_{j m} \operatorname{Tr}\left(c_{j}^{\dagger} c_{n} \rho\right) \\
& =\sum_{k}\left(-i h_{n k} \Delta_{m k}+i h_{k m} \Delta_{k n}\right),
\end{aligned}
$$

the second term gives

$$
\begin{aligned}
\sum_{\mu} \operatorname{Tr}\left(2 L_{\mu}^{\dagger}\left[c_{m}^{\dagger} c_{n}, L_{\mu}\right] \rho\right) & =\sum_{\mu} \operatorname{Tr}\left(2 L_{\mu}^{g \dagger}\left[c_{m}^{\dagger} c_{n}, L_{\mu}^{g}\right] \rho+2 L_{\mu}^{l \dagger}\left[c_{m}^{\dagger} c_{n}, L_{\mu}^{l}\right] \rho\right) \\
& =\sum_{\mu j k} \operatorname{Tr}\left(2 D_{\mu j}^{g *} D_{\mu k}^{g} c_{j}\left[c_{m}^{\dagger} c_{n}, c_{k}^{\dagger}\right] \rho+2 D_{\mu j}^{l *} D_{\mu k}^{l} c_{j}^{\dagger}\left[c_{m}^{\dagger} c_{n}, c_{k}\right] \rho\right) \\
& =\sum_{\mu j k} \operatorname{Tr}\left(2 D_{\mu j}^{g *} D_{\mu k}^{g} c_{j} c_{m}^{\dagger}\left\{c_{n}, c_{k}^{\dagger}\right\} \rho-2 D_{\mu j}^{l *} D_{\mu k}^{l} c_{j}^{\dagger}\left\{c_{k}, c_{m}^{\dagger}\right\} c_{n} \rho\right) \\
& =\sum_{\mu j k} \operatorname{Tr}\left(2 \delta_{n, k} D_{\mu j}^{g *} D_{\mu k}^{g} c_{j} c_{m}^{\dagger} \rho-2 \delta_{k, m} D_{\mu j}^{l *} D_{\mu k}^{l} c_{j}^{\dagger} c_{n} \rho\right) \\
& =\sum_{\mu j} \operatorname{Tr}\left(2 D_{\mu j}^{g *} D_{\mu n}^{g} c_{j} c_{m}^{\dagger} \rho-2 D_{\mu j}^{l *} D_{\mu m}^{l} c_{j}^{\dagger} c_{n} \rho\right) \\
& =\sum_{j} 2 M_{j n}^{g}\left(\delta_{m j}-\Delta_{m j}\right)-2 M_{j m}^{l} \Delta_{j n} \\
& =2 M_{m n}^{g}-\sum_{j}\left(2 M_{j n}^{g} \Delta_{m j}+2 M_{j m}^{l} \Delta_{j n}\right),
\end{aligned}
$$

and the third term gives

$$
\begin{aligned}
& \sum_{\mu} \operatorname{Tr}\left(\left[L_{\mu}^{\dagger} L_{\mu}, c_{m}^{\dagger} c_{n}\right] \rho\right) \\
& \quad=\sum_{\mu j k} \operatorname{Tr}\left(D_{\mu j}^{g *} D_{\mu k}^{g}\left[c_{j} c_{k}^{\dagger}, c_{m}^{\dagger} c_{n}\right] \rho+D_{\mu j}^{l *} D_{\mu k}^{l}\left[c_{j}^{\dagger} c_{k}, c_{m}^{\dagger} c_{n}\right] \rho\right) \\
& =\sum_{\mu j k} \operatorname{Tr}\left[D_{\mu j}^{g *} D_{\mu k}^{g}\left(-\left\{c_{j}, c_{m}^{\dagger}\right\} c_{k}^{\dagger} c_{n}+c_{m}^{\dagger} c_{j}\left\{c_{k}^{\dagger}, c_{n}\right\}\right) \rho+D_{\mu j}^{l *} D_{\mu k}^{l}\left(c_{j}^{\dagger}\left\{c_{k}, c_{m}^{\dagger}\right\} c_{n}-c_{m}^{\dagger}\left\{c_{n}, c_{j}^{\dagger}\right\} c_{k}\right) \rho\right] \\
& =\sum_{j k} \operatorname{Tr}\left[M_{j k}^{g}\left(-\delta_{j, m} c_{k}^{\dagger} c_{n}+\delta_{k, n} c_{m}^{\dagger} c_{j}\right) \rho+M_{j k}^{l}\left(\delta_{k, m} c_{j}^{\dagger} c_{n}-\delta_{j, n} c_{m}^{\dagger} c_{k}\right) \rho\right]
\end{aligned}
$$




$$
\begin{aligned}
& =\operatorname{Tr}\left[\sum_{k}-M_{m k}^{g} c_{k}^{\dagger} c_{n} \rho+\sum_{j} M_{j n}^{l} c_{m}^{\dagger} c_{j} \rho+\sum_{j} M_{j m}^{l} c_{j}^{\dagger} c_{n} \rho-\sum_{k} M_{n k}^{l} c_{m}^{\dagger} c_{k} \rho\right] \\
& =\sum_{k}\left(-M_{m k}^{g} \Delta_{k n}+M_{k n}^{g} \Delta_{m k}+M_{k m}^{l} \Delta_{k n}-M_{n k}^{l} \Delta_{m k}\right) .
\end{aligned}
$$

Combining them together, we get

$$
\begin{aligned}
\left(\frac{d \Delta_{m n}}{d t}\right) & =2 M_{m n}^{g}+\sum_{k}\left(-i h_{n k} \Delta_{m k}+i h_{k m} \Delta_{k n}-2 M_{k n}^{g} \Delta_{m k}-2 M_{k m}^{l} \Delta_{k n}-M_{m k}^{g} \Delta_{k n}+M_{k n}^{g} \Delta_{m k}+M_{k m}^{l} \Delta_{k n}-M_{n k}^{l} \Delta_{m k}\right) \\
& =2 M_{m n}^{g}+\sum_{k}\left(-i h_{n k} \Delta_{m k}+i h_{k m} \Delta_{k n}-M_{k n}^{g} \Delta_{m k}-M_{k m}^{l} \Delta_{k n}-M_{m k}^{g} \Delta_{k n}-M_{n k}^{l} \Delta_{m k}\right) \\
& =\left(2 M^{g}+i\left[h^{T}, \Delta\right]-\left\{M^{g}+M^{l T}, \Delta\right\}\right)_{m n} \\
& =\left(2 M^{g}+X \Delta+\Delta X^{\dagger}\right)_{m n},
\end{aligned}
$$

where

$$
X=i h^{T}-\left(M^{g}+M^{l T}\right) .
$$

For $h$ and $L_{\mu}$ given by Eqs. (2) and (3) in the main text, we can calculate that $M^{g}=\frac{\gamma_{z}}{2}\left(\sigma_{0} \tau_{0}-\sigma_{y} \tau_{z}\right), M^{l}=\frac{\gamma_{l}}{2}\left(\sigma_{0} \tau_{0}+\sigma_{y} \tau_{z}\right)$, and $X=i t_{1} \sigma_{x}+i\left(t_{2} \sigma_{y}+\delta_{1} \tau_{x}\right) \sin k+i\left(t_{2} \sigma_{x}+\delta_{2} \sigma_{y} \tau_{x}\right) \cos k-\frac{\gamma_{l}+\gamma_{g}}{2}\left(\sigma_{0} \tau_{0}-\sigma_{y} \tau_{z}\right)$, which gives Eq. (5) in the main text. Let $\frac{d \Delta}{d t}=2 M^{g}+X \Delta+\Delta X^{\dagger}=0$, and the solution is $\Delta_{s}=\frac{\gamma_{g}}{\gamma} \mathbb{I}$. We can verify that $2 M^{g}+X \Delta_{s}+\Delta_{s} X^{\dagger}=\gamma_{g}\left(\sigma_{0} \tau_{0}-\sigma_{y} \tau_{z}\right)-$ $2 \frac{\gamma_{g}}{\gamma} \frac{\gamma_{l}+\gamma_{g}}{2}\left(\sigma_{0} \tau_{0}-\sigma_{y} \tau_{z}\right)=0$

\section{APPENDIX B: EXAMPLE WITH $Z_{2}$ SKIN EFFECT BUT NO HELICAL DAMPING AND GENERALIZED HELICAL DAMPING}

In this Appendix, we show that $\mathbb{Z}_{2}$ skin effect is not a sufficient condition for the occurrence of helical damping of particle number. When particle number damping in the periodic boundary system fulfills an exponential law, the particle number damping in the open boundary system always follows an exponential law. In this case, the Liouvillian gap is not zero. Consider a 1D lattice with each cell having one orbit and spin degree of freedom, described by the following Hamiltonian in the momentum space:

$$
h(k)=2 t_{1} \cos k+2 \delta \tau_{x} \sin k,
$$

where $\tau_{\alpha}(\alpha=x, y, z)$ act on the spin space. Suppose that the Lindblad operators are given by

$$
\begin{aligned}
& L_{x 1}^{g}=\sqrt{\frac{\gamma_{g}}{2}}\left(c_{x \uparrow}^{\dagger}-c_{x \downarrow}^{\dagger}+i c_{x+1 \uparrow}^{\dagger}+i c_{x+1 \downarrow}^{\dagger}\right), \\
& L_{x 2}^{g}=\sqrt{\frac{\gamma_{g}}{2}}\left(c_{x \uparrow}^{\dagger}+c_{x \downarrow}^{\dagger}+i c_{x+1 \uparrow}^{\dagger}-i c_{x+1 \downarrow}^{\dagger}\right) .
\end{aligned}
$$

It follows $\tilde{\Delta}(t)=\Delta(t)-\Delta_{s}=e^{X t} \tilde{\Delta}(0) e^{X^{\dagger} t}$, where $X=2 i t_{1} \cos k-2 \gamma_{g} \tau_{0}+\left(2 i \delta \tau_{x}+2 \gamma_{g} \tau_{z}\right) \sin k$ and $\Delta_{s}=\mathbb{I}$. We display the spectrum of the $X$ matrix under PBC and OBC in Fig. 7, which indicates the existence of nonzero Liouvillian gap for both periodic and open boundary systems. The disappearance of skin modes, after putting two identical models together and adding a small symmetry-allowed perturbation, indicates the existence of $\mathbb{Z}_{2}$ skin effect.

Set the initial state as the state without particle occupation, and we can get $\tilde{\Delta}=-\mathbb{I}$. We show the relative local particle number damping for different cells in Fig. 8(a). The evolution of $\left|\tilde{n}_{x}(t)\right|$ under PBC and OBC are displayed in Figs. 8(b) and 8(c), respectively. It is shown that the particle number damping under both $\mathrm{PBC}$ and $\mathrm{OBC}$ fulfills exponential law. In Fig. 8(d), we display the evolution of $\frac{\left(\left|\tilde{n}_{x}(t)\right|_{\mathrm{OBC}}\right.}{\left(\tilde{n}_{x}(t) \mid{ }_{\mathrm{PBC}}\right.}$, which exhibits helical behavior. And we call it generalized helical damping. The generalized helical damping is a more inclusive physical phenomenon than helical damping, and it does not need the Liouvillian gap under PBC to be gapless. Instead, the mismatch of the open and periodic boundary damping spectrum is the necessary condition for helical damping.

\section{APPENDIX C: THE DECAY FACTOR OF RELATIVE PARTICLE NUMBER}

The $k+i \kappa$ component relative particle number in each cell is

$$
\begin{aligned}
\tilde{n}_{x, k+i \kappa} & =\sum_{s, o}\left\langle(x, s, o)\left|e^{X(k+i \kappa) t} e^{X^{\dagger}(k+i \kappa) t}\right|(x, s, o)\right\rangle \\
& =\sum_{s, o, x_{1}, s_{1}, o_{1}}\left\langle(x, s, o)\left|e^{X(k+i \kappa) t}\right|\left(x_{1}, s_{1}, o_{1}\right)\right\rangle\left\langle\left(x_{1}, s_{1}, o_{1}\right)\left|e^{X^{\dagger}(k+i \kappa) t}\right|(x, s, o)\right\rangle
\end{aligned}
$$




$$
\begin{aligned}
& =\sum_{s, o, x_{1}, s_{1}, o_{1}}\left|\left\langle(x, s, o)\left|e^{X(k+i \kappa) t}\right|\left(x_{1}, s_{1}, o_{1}\right)\right\rangle\right|^{2} \\
& =\sum_{\alpha} \sum_{s, o, x_{1}, s_{1}, o_{1}}\left|\left\langle(x, s, o)\left|e^{X(k+i \kappa) t}\right| k+i \kappa, \alpha\right\rangle_{R L}\left\langle k+i \kappa, \alpha \mid\left(x_{1}, s_{1}, o_{1}\right)\right\rangle\right|^{2} \\
& =\sum_{\alpha} \sum_{s, o, x_{1}, s_{1}, o_{1}}\left|\langle(s, o) \mid \alpha\rangle_{R L}\left\langle\alpha \mid\left(s_{1}, o_{1}\right)\right\rangle e^{i(k+i \kappa)\left(x-x_{1}\right)+\lambda_{k+i k, \alpha} t}\right|^{2} \propto \sum_{\alpha} \sum_{s, o, x_{1}, s_{1}, o_{1}}\left|e^{i(k+i \kappa)\left(x_{1}-x\right)+\lambda_{k+i \kappa, \alpha} t}\right|^{2},
\end{aligned}
$$

and

$$
\max \left\{\tilde{n}_{x, k+i \kappa}\right\} \propto \max \left\{\sum_{\alpha} \sum_{s, o, x_{1}, s_{1}, o_{1}}\left|e^{i(k+i \kappa)\left(x-x_{1}\right)+\lambda_{k+i \kappa, \alpha} t}\right|^{2}\right\} \propto\left|e^{2 i(k+i \kappa)\left(x-x_{1}\right)-\gamma t}\right| \propto e^{2 \kappa\left(x_{1}-x\right)-\gamma t},
$$

where $\alpha$ is the band index, $\lambda_{k+i \kappa, \alpha}\left(|k+i \kappa, \alpha\rangle_{R}\right)$ is the eigenvalue (eigenvector) of $X(k+i \kappa)$ corresponding to the $\alpha$ band, $\max \left\{\operatorname{Re}\left(\lambda_{k+i \kappa, \alpha}\right)\right\}=-\frac{\gamma}{2},\langle x \mid k+i \kappa\rangle_{R}=e^{i(k+i \kappa) x}$, and ${ }_{L}\left\langle k+i \kappa \mid x_{1}\right\rangle=e^{-i(k+i \kappa) x_{1}}$.

For parameter values $t_{1}=t_{2}=1, \gamma=0.4, \delta_{1}=0.1$, and $\delta_{2}=0$, we illustrate the GBZ, $\operatorname{Re}(i \lambda)$ versus $k$ and $v$ as a function of $k$ in Figs. 9(a)-9(c), respectively. We can get $v_{\max }=\max \left\{\operatorname{Re}\left(\frac{i \partial \lambda_{k+i k, \alpha}}{\partial k}\right)\right\} \approx 1, v_{\min }=\min \left\{\operatorname{Re}\left(\frac{i \partial \lambda_{k+i k, \alpha}}{\partial k}\right)\right\} \approx-1, \kappa_{\max }=0.2$, and $\kappa_{\min }=-0.2$ at $k=\pi$. There is an accidental symmetry $\sigma_{z} \tau_{z}\left(X+\frac{\gamma}{2}\right)=-\left(X+\frac{\gamma}{2}\right) \sigma_{z} \tau_{z}$, which protects $v_{\max }=-v_{\min }$.

Furthermore, if $\delta_{1}, \delta_{2}, \frac{\gamma}{t_{1}} \ll 1$,

$$
X \approx i\left[\begin{array}{cc}
H_{\mathrm{nSSH}}(k)+\frac{i \gamma}{2} & 0 \\
0 & H_{\mathrm{nSSH}}^{T}(-k)+\frac{i \gamma}{2}
\end{array}\right]=\left(-\frac{\gamma}{2}+i t_{1} \sigma_{x}+\frac{\gamma}{2} \sigma_{y} \tau_{z}\right)+i t_{2} \sigma_{y} \sin k+i t_{2} \sigma_{x} \cos k .
$$

The eigenvalues of open boundary $X$ matrix are

$$
\lambda_{k+i \kappa, \alpha=1,2,3,4}=-\frac{\gamma}{2} \pm i \sqrt{t_{1}^{2}+t_{2}^{2}+2 t_{1} t_{2} \cos (k+i \kappa)-\frac{\gamma^{2}}{4} \pm i \gamma t_{2} \sin (k+i \kappa)} .
$$

We can get $v_{\max }=-v_{\min } \approx \min \left\{t_{1}, t_{2}\right\}$ and $\kappa_{\max }=-\kappa_{\min } \approx \frac{\gamma}{2 t_{1}}$ at $k=\pi$. So helical damping still exists for $\delta_{1}, \delta_{2}, \frac{\gamma}{t_{1}} \ll 1$, and $t_{1}<t_{2}$.

\section{APPENDIX D: PROVE THE EQUIVALENCE OF GBZ EQUATIONS FOR CASE 1 AND CASE 3}

For case 1 , the GBZ equations are $\left|z_{1}^{a}\right|=\left|z_{2}^{a}\right|$ and $\left|z_{1}^{b}\right|=\left|z_{2}^{b}\right|$, where $\left|z_{1}^{a}\right| \leqslant\left|z_{2}^{a}\right|$ and $z_{i}^{b}=\frac{1}{z_{i}^{a}}(i=1,2)$. Without loss of generality, we assume that $\left|z_{2}^{a}\right| \leqslant 1$. Then the roots of $f(z)=0$ are $z_{1}^{a}, z_{2}^{a}, z_{1}^{b}, z_{2}^{b}$ with $\left|z_{1}^{a}\right| \leqslant\left|z_{2}^{a}\right| \leqslant\left|z_{2}^{b}\right| \leqslant\left|z_{1}^{b}\right|$, and GBZ equations are $\left|z_{1}^{a}\right|=\left|z_{2}^{a}\right|$ and $\left|z_{1}^{b}\right|=\left|z_{2}^{b}\right|$, where $z_{i}^{b}=\frac{1}{z_{i}^{a}}$. And it is the GBZ equation of case 3 .

[1] N. Hatano and D. R. Nelson, Localization Transitions in Non-Hermitian Quantum Mechanics, Phys. Rev. Lett. 77, 570 (1996).

[2] M. S. Rudner and L. S. Levitov, Topological Transition in a Non-Hermitian Quantum Walk, Phys. Rev. Lett. 102, 065703 (2009).

[3] K. Esaki, M. Sato, K. Hasebe, and M. Kohmoto, Edge states and topological phases in non-Hermitian systems, Phys. Rev. B 84, 205128 (2011).

[4] T. E. Lee, Anomalous Edge State in a Non-Hermitian Lattice, Phys. Rev. Lett. 116, 133903 (2016).

[5] D. Leykam, K. Y. Bliokh, C. Huang, Y. D. Chong, and F. Nori, Edge Modes, Degeneracies, and Topological Numbers in Non-Hermitian Systems, Phys. Rev. Lett. 118, 040401 (2017).

[6] H. Shen, B. Zhen, and L. Fu, Topological Band Theory for Non-Hermitian Hamiltonians, Phys. Rev. Lett. 120, 146402 (2018)

[7] Y. Xu, S.-T. Wang, and L.-M. Duan, Weyl Exceptional Rings in a Three-Dimensional Dissipative Cold Atomic Gas, Phys. Rev. Lett. 118, 045701 (2017).
[8] Z. Gong, Y. Ashida, K. Kawabata, K. Takasan, S. Higashikawa, and M. Ueda, Topological Phases of Non-Hermitian Systems, Phys. Rev. X 8, 031079 (2018).

[9] C. Yin, H. Jiang, L. Li, R. Lü, and S. Chen, Geometrical meaning of winding number and its characterization of topological phases in one-dimensional chiral non-Hermitian systems, Phys. Rev. A 97, 052115 (2018).

[10] S. Lieu, Topological phases in the non-Hermitian Su-SchriefferHeeger model, Phys. Rev. B 97, 045106 (2018).

[11] V. M. Martinez Alvarez, J. E. Barrios Vargas, and L. E. F. Foa Torres, Non-Hermitian robust edge states in one dimension: Anomalous localization and eigenspace condensation at exceptional points, Phys. Rev. B 97, 121401(R) (2018).

[12] Y. Xiong, Why does bulk boundary correspondence fail in some non-Hermitian topological models, J. Phys. Commun. 2, 035043 (2018).

[13] S. Yao and Z. Wang, Edge States and Topological Invariants of Non-Hermitian Systems, Phys. Rev. Lett. 121, 086803 (2018).

[14] F. K. Kunst, E. Edvardsson, J. C. Budich, and E. J. Bergholtz, Biorthogonal Bulk-Boundary Correspondence in Non-Hermitian Systems, Phys. Rev. Lett. 121, 026808 (2018). 
[15] S. Yao, F. Song, and Z. Wang, Non-Hermitian Chern Bands, Phys. Rev. Lett. 121, 136802 (2018).

[16] C.-H. Liu, H. Jiang, and S. Chen, Topological classification of non-Hermitian systems with reflection symmetry, Phys. Rev. B 99, 125103 (2019).

[17] K. Kawabata, K. Shiozaki, M. Ueda, and M. Sato, Symmetry and Topology in Non-Hermitian Physics, Phys. Rev. X 9, 041015 (2019).

[18] H. Zhou and J. Y. Lee, Periodic table for topological bands with non-Hermitian symmetries, Phys. Rev. B 99, 235112 (2019).

[19] C.-H. Liu, and S. Chen, Topological classification of defects in non-Hermitian systems, Phys. Rev. B 100, 144106 (2019).

[20] F. Song, S. Yao, and Z. Wang, Non-Hermitian Topological Invariants in Real Space, Phys. Rev. Lett. 123, 246801 (2019).

[21] T. Yoshida, T. Mizoguchi, and Y. Hatsugai, Mirror skin effect and its electric circuit simulation, Phys. Rev. Research 2, 022062 (2020).

[22] T. Ozawa, H. M. Price, A. Amo, N. Goldman, M. Hafezi, L. Lu, M. Rechtsman, D. Schuster, J. Simon, O. Zilberberg, and I. Carusotto, Topological photonics, Rev. Mod. Phys. 91, 015006 (2019).

[23] A. Guo, G. J. Salamo, D. Duchesne, R. Morandotti, M. VolatierRavat, V. Aimez, G. A. Siviloglou, and D. N. Christodoulides, Observation of PT-Symmetry Breaking in Complex Optical Potentials, Phys. Rev. Lett. 103, 093902 (2009).

[24] B. Peng, S. K. Ozdemir, F. Lei, F. Monifi, M. Gianfreda, G. L. Long, S. Fan, F. Nori, C. M. Bender, and L. Yang, Parity-timesymmetric whispering-gallery microcavities, Nat. Phys. 10, 394 (2014).

[25] G. Harari, M. A. Bandres, Y. Lumer, M. C. Rechtsman, Y. D. Chong, M. Khajavikhan, D. N. Christodoulides, and M. Segev, Topological insulator laser: Theory, Science 359, eaar4003 (2018).

[26] S. Diehl, A. Micheli, A. Kantian, B. Kraus, H. P. Büchler, and P. Zoller, Quantum states and phases in driven open quantum systems with cold atoms, Nat. Phys. 4, 878 (2008).

[27] I. Rotter, A non-Hermitian Hamilton operator and the physics of open quantum systems, J. Phys. A 42, 153001 (2009).

[28] H. J. Carmichael, Quantum Trajectory Theory for Cascaded Open Systems, Phys. Rev. Lett. 70, 2273 (1993).

[29] G. Lindblad, On the generators of quantum dynamical semigroups, Commun. Math. Phys. 48, 119 (1976).

[30] V. Gorini, A. Kossakowski, and E. C. G. Sudarshan, Completely positive dynamical semigroups of N-level systems, J. Math. Phys. 17, 821 (1976).

[31] T. Prosen, Third quantization: A general method to solve master equations for quadratic open Fermi systems, New J. Phys. 10, 043026 (2008).

[32] T. Prosen, Spectral theorem for the Lindblad equation for quadratic open fermionic systems, J. Stat. Mech. (2010) P07020.

[33] B. Zhu, R. Lü, and S. Chen, PT symmetry in the non-Hermitian Su-Schrieffer-Heeger model with complex boundary potentials, Phys. Rev. A 89, 062102 (2014).

[34] C. Yuce, Majorana edge modes with gain and loss, Phys. Rev. A 93, 062130 (2016).

[35] H. Menke and M. M. Hirschmann, Topological quantum wires with balanced gain and loss, Phys. Rev. B 95, 174506 (2017).
[36] F. Dangel, M. Wagner, H. Cartarius, J. Main, and G. Wunner, Topological invariants in dissipative extensions of the SuSchrieffer-Heeger model, Phys. Rev. A 98, 013628 (2018).

[37] D. S. Borgnia, A. J. Kruchkov, and R.-J. Slager, Non-Hermitian Boundary Modes, Phys. Rev. Lett. 124, 056802 (2020).

[38] X. R. Wang, C. X. Guo, and S. P. Kou, Defective edge states and number-anomalous bulk-boundary correspondence in nonHermitian topological systems, Phys. Rev. B 101, 121116(R) (2020).

[39] R. Chen, C.-Z. Chen, B. Zhou, and D.-H. Xu, Finite-size effects in non-Hermitian topological systems, Phys. Rev. B 99, 155431 (2019).

[40] T. Liu, Yu-R. Zhang, Q. Ai, Z. Gong, K. Kawabata, M. Ueda, and F. Nori, Second-Order Topological Phases in NonHermitian Systems, Phys. Rev. Lett. 122, 076801 (2019).

[41] S. Diehl, E. Rico, M. A. Baranov, and P. Zoller, Topology by dissipation in atomic quantum wires, Nat. Phys. 7, 971 (2011).

[42] T. Prosen and E. Ilievski, Nonequilibrium Phase Transition in a Periodically Driven xy Spin Chain, Phys. Rev. Lett. 107, 060403 (2011).

[43] C. E. Bardyn, M. A. Baranov, C. V. Kraus, E. Rico, A Imamoğlu, P. Zoller, and S. Diehl, Topology by dissipation, New J. Phys. 15, 085001 (2013).

[44] M. Goldstein, Dissipation-induced topological insulators: A nogo theorem and a recipe, SciPost Phys. 7, 067 (2019).

[45] M. van Caspel, S. E. T. Arze, and I. P. Castillo, Dynamical signatures of topological order in the driven-dissipative Kitaev chain, SciPost Phys. 6, 26 (2019).

[46] M. J. Kastoryano and M. S. Rudner, Topological transport in the steady state of a quantum particle with dissipation, Phys. Rev. B 99, 125118 (2019).

[47] F. Song, S. Yao, and Z. Wang, Non-Hermitian Skin Effect and Chiral Damping in Open Quantum Systems, Phys. Rev. Lett. 123, 170401 (2019).

[48] S. Lieu, M. McGinley, and N. R. Cooper, Tenfold Way for Quadratic Lindbladians, Phys. Rev. Lett. 124, 040401 (2020).

[49] C. H. Lee and R. Thomale, Anatomy of skin modes and topology in non-Hermitian systems, Phys. Rev. B 99, 201103(R) (2019).

[50] L. Herviou, J. H. Bardarson, and N. Regnault, Defining a bulk-edge correspondence for non-Hermitian Hamiltonians via singular-value decomposition, Phys. Rev. A 99, 052118 (2019).

[51] T.-S. Deng and W. Yi, Non-Bloch topological invariants in a non-Hermitian domain wall system, Phys. Rev. B 100, 035102 (2019).

[52] H. Jiang, L.-J. Lang, C. Yang, S.-L. Zhu, and S. Chen, Interplay of non-Hermitian skin effects and Anderson localization in nonreciprocal quasiperiodic lattices, Phys. Rev. B 100, 054301 (2019).

[53] L. Jin and Z. Song, Bulk-boundary correspondence in nonhermitian systems, Phys. Rev. B 99, 081103(R) (2019).

[54] C. H. Lee, L. Li, and J. Gong, Hybrid Higher-Order SkinTopological Modes in Nonreciprocal Systems, Phys. Rev. Lett. 123, 016805 (2019).

[55] S. Longhi, Probing non-Hermitian skin effect and non-Bloch phase transitions, Phys. Rev. Research 1, 023013 (2019)

[56] F. K. Kunst and V. Dwivedi, Non-Hermitian systems and topology: A transfer-matrix perspective, Phys. Rev. B 99, 245116 (2019). 
[57] C. H. Lee, L. Li, R. Thomale, and J. Gong, Unraveling non-Hermitian pumping: Emergent spectral singularities and anomalous responses, Phys. Rev. B 102, 085151 (2020).

[58] N. Okuma and M. Sato, Topological Phase Transition Driven by Infinitesimal Instability: Majorana Fermions in Non-Hermitian Spintronics, Phys. Rev. Lett. 123, 097701 (2019).

[59] L. Xiao, T. Deng, K. Wang, G. Zhu, Z. Wang, W. Yi, and P. Xue, Non-Hermitian bulk-boundary correspondence in quantum dynamics, Nat. Phys. 16, 761 (2020).

[60] T. Helbig, T. Hofmann, S. Imhof, M. Abdelghany, T. Kiessling, L. W. Molenkamp, C. H. Lee, A. Szameit, M. Greiter, and R. Thomale, Generalized bulk-boundary correspondence in non-Hermitian topolectrical circuits, Nat. Phys. 16, 747 (2020).

[61] A. Ghatak, M. Brandenbourger, J. van Wezel, and C. Coulais, Observation of non-Hermitian topology and its bulk-edge correspondence, arXiv:1907.11619.

[62] K. Yokomizo and S. Murakami, Non-Bloch Band Theory of Non-Hermitian Systems, Phys. Rev. Lett. 123, 066404 (2019).
[63] K. Zhang, Z. Yang, and C. Fang, Correspondence between Winding Numbers and Skin Modes in Non-Hermitian Systems, Phys. Rev. Lett. 125, 126402 (2020).

[64] Z. Yang, K. Zhang, C. Fang, and J. P. Hu, Auxiliary generalized Brillouin zone method in non-Hermitian band theory, arXiv:1912.05499.

[65] Y. Yi and Z. Yang, Non-Hermitian skin modes induced by onsite dissipations and chiral tunneling effect, arXiv:2003.2219.

[66] N. Okuma, K. Kawabata, K. Shiozaki, and M. Sato, Topological Origin of Non-Hermitian Skin Effects, Phys. Rev. Lett. 124, 086801 (2020).

[67] L. Li, C. H. Lee, S. Mu, and J. Gong, Critical non-Hermitian skin effect, arXiv:2003.3039.

[68] X. Zhu, H. Wang, S. K. Gupta, H. Zhang, B. Xie, M. Lu, and Y. Chen, Photonic non-Hermitian skin effect and non-Bloch bulk-boundary correspondence, Phys. Rev. Research 2, 013280 (2020).

[69] T. Hofmann, T. Helbig, F. Schindler, N. Salgo, M. Brzezinska, M. Greiter, T. Kiessling, D. Wolf, A. Vollhardt, A. Kabasi, C. H. Lee, A. Bilusic, R. Thomale, and T. Neupert, Reciprocal skin effect and its realization in a topolectrical circuit, Phys. Rev. Research 2, 023265 (2020). 